Georgian Mathematical Journal

Volume 11 (2004), Number 1, 49-58

\title{
EXPLICIT SOLUTIONS OF THE BASIC BOUNDARY VALUE PROBLEMS OF STATICS OF THE ELASTIC MIXTURE THEORY FOR AN ANNULUS
}

\author{
M. BASHELEISHVILI
}

\begin{abstract}
Using the complex representation formulae of regular solutions of equations of statics of the theory of elastic mixtures, we construct the explicit solutions of the Dirichlet and Neumann type boundary value problems for an annulus in the form of absolutely and uniformly convergent series.
\end{abstract}

2000 Mathematics Subject Classification: 74B05.

Key words and phrases: Elastic mixture, elasticity.

\section{Basic Equations and Some Auxiliary Questions}

The basic homogeneous equations of statics of the elastic mixture theory are written in terms of displacement components as follows [1]:

$$
\begin{aligned}
& a_{1} \Delta u^{\prime}+b_{1} \text { grad div } u^{\prime}+c \Delta u^{\prime \prime}+d \operatorname{grad} \operatorname{div} u^{\prime \prime}=0, \\
& c \Delta u^{\prime}+d \operatorname{grad} \operatorname{div} u^{\prime}+a_{2} \Delta u^{\prime \prime}+b_{2} \operatorname{grad} \operatorname{div} u^{\prime \prime}=0 ;
\end{aligned}
$$

here $\Delta$ is a two-dimensional Laplace operator, $u^{\prime}=\left(u_{1}^{\prime}, u_{2}^{\prime}\right)$ and $u^{\prime \prime}=\left(u_{1}^{\prime \prime}, u_{2}^{\prime \prime}\right)$ are partial displacements,

$$
\begin{aligned}
& a_{1}=\mu_{1}-\lambda_{5}, \quad a_{2}=\mu_{2}-\lambda_{5}, \quad c=\mu_{3}+\lambda_{5}, \\
& b_{1}=\mu_{1}+\lambda_{1}+\lambda_{5}-\frac{\alpha_{2}}{\rho_{*}} \rho_{2}, \quad b_{2}=\mu_{2}+\lambda_{2}+\lambda_{5}+\frac{\alpha_{2}}{\rho_{*}} \rho_{1}, \\
& d=\mu_{3}+\lambda_{3}-\lambda_{5}-\frac{\alpha_{2}}{\rho_{*}} \rho_{1} \equiv \mu_{3}+\lambda_{4}-\lambda_{5} \frac{\alpha_{2}}{\rho_{*}} \rho_{2}, \\
& \rho_{*}=\rho_{1}+\rho_{2}, \quad \alpha_{2}=\lambda_{3}-\lambda_{4},
\end{aligned}
$$

where $\mu_{1}, \mu_{2}, \mu_{3}, \lambda_{1}, \lambda_{2}, \lambda_{3}, \lambda_{4}, \lambda_{5}, \rho_{1}, \rho_{2}$ are the constants characterizing the physical properties of a mixture and satisfying certain inequalities [2].

System (1.1) can be rewritten as

$$
\begin{aligned}
& a_{1} \Delta u^{\prime}+c \Delta u^{\prime \prime}+b_{1} \operatorname{grad} \theta^{\prime}+d \operatorname{grad} \theta^{\prime \prime}=0, \\
& c \Delta u^{\prime}+a_{2} \Delta u^{\prime \prime}+d \operatorname{grad} \theta^{\prime}+b_{2} \operatorname{grad} u^{\prime \prime}=0,
\end{aligned}
$$

where

$$
\theta^{\prime}=\frac{\partial u_{1}^{\prime}}{\partial x_{1}}+\frac{\partial u_{2}^{\prime}}{\partial x_{2}}, \quad \theta^{\prime \prime}=\frac{\partial u_{1}^{\prime \prime}}{\partial x_{1}}+\frac{\partial u_{2}^{\prime \prime}}{\partial x_{2}}
$$


Introducing the variables $z=x_{1}+i x_{2}, \bar{z}=x_{1}-i x_{2}$, where $x_{1}=\frac{z+\bar{z}}{2}, x_{2}=\frac{z-\bar{z}}{2 i}$, we have

$$
\begin{gathered}
\frac{\partial}{\partial x_{1}}=\frac{\partial}{\partial z}+\frac{\partial}{\partial \bar{z}}, \quad \frac{\partial}{\partial x_{2}}=i\left(\frac{\partial}{\partial z}-\frac{\partial}{\partial \bar{z}}\right) \\
\frac{\partial}{\partial z}=\frac{1}{2}\left(\frac{\partial}{\partial x_{1}}-i \frac{\partial}{\partial x_{2}}\right), \quad \frac{\partial}{\partial \bar{z}}=\frac{1}{2}\left(\frac{\partial}{\partial x_{1}}+i \frac{\partial}{\partial x_{2}}\right) .
\end{gathered}
$$

By elementary calculations we obtain

$$
\Delta=4 \frac{\partial^{2}}{\partial z \partial \bar{z}}, \quad \theta^{\prime}=\frac{\partial U_{1}}{\partial z}+\frac{\partial \bar{U}_{1}}{\partial \bar{z}}, \quad \theta^{\prime \prime}=\frac{\partial U_{2}}{\partial z}+\frac{\partial \bar{U}_{2}}{\partial \bar{z}},
$$

where

$$
\begin{array}{cl}
U_{1}=u_{1}+i u_{2}, & U_{2}=u_{3}+i u_{4}, \\
u_{1}=u_{1}^{\prime}, \quad u_{2}=u_{2}^{\prime}, \quad u_{3}=u_{1}^{\prime \prime}, \quad u_{4}=u_{2}^{\prime \prime} .
\end{array}
$$

Taking into account formulas (1.4), (1.5), and (1.6), we rewrite system (1.2) in the complex form

$$
\begin{aligned}
& 2 a_{1} \frac{\partial^{2} U_{1}}{\partial z \partial \bar{z}}+2 c \frac{\partial^{2} U_{2}}{\partial z \partial \bar{z}}+b_{1} \frac{\partial \theta^{\prime}}{\partial \bar{z}}+d \frac{\partial \theta^{\prime \prime}}{\partial \bar{z}}=0 \\
& 2 c \frac{\partial^{2} U_{1}}{\partial z \partial \bar{z}}+2 a_{2} \frac{\partial^{2} U_{2}}{\partial z \partial \bar{z}}+d \frac{\partial \theta^{\prime}}{\partial \bar{z}}+b_{2} \frac{\partial \theta^{\prime \prime}}{\partial \bar{z}}=0 .
\end{aligned}
$$

Using (1.5), we find

$$
\begin{aligned}
& \left(2 a_{1}+b_{1}\right) \frac{\partial^{2} U_{1}}{\partial z \partial \bar{z}}+(2 c+d) \frac{\partial^{2} U_{2}}{\partial z \partial \bar{z}}+b_{1} \frac{\partial^{2} \bar{U}_{1}}{\partial \bar{z}^{2}}+d \frac{\partial^{2} \bar{U}_{2}}{\partial \bar{z}^{2}}=0 \\
& (2 c+d) \frac{\partial^{2} U_{1}}{\partial z \partial \bar{z}}+\left(2 a_{2}+b_{2}\right) \frac{\partial^{2} U_{2}}{\partial z \partial \bar{z}}+d \frac{\partial^{2} \bar{U}_{1}}{\partial \bar{z}^{2}}+b_{2} \frac{\partial^{2} \bar{U}_{2}}{\partial \bar{z}^{2}}=0
\end{aligned}
$$

from which we obtain

$$
\frac{\partial^{2} U}{\partial z \partial \bar{z}}+\varepsilon^{T} \frac{\partial^{2} \bar{U}}{\partial \bar{z}^{2}}
$$

where $U=\left(U_{1}, U_{2}\right)=\left(u_{1}+i u_{2}, u_{3}+i u_{4}\right), U_{1}$ and $U_{2}$ are defined by (1.6),

$$
\begin{aligned}
\varepsilon^{T} & =\left[\begin{array}{ll}
\varepsilon_{1}, & \varepsilon_{3} \\
\varepsilon_{2}, & \varepsilon_{4}
\end{array}\right], \\
\delta_{0} \varepsilon_{1} & =2\left(a_{2} b_{1}-c d\right)+b_{1} b_{2}-d^{2}, \quad \delta_{0} \varepsilon_{2}=2\left(d a_{1}-c b_{1}\right), \\
\delta_{0} \varepsilon_{3} & =2\left(d a_{2}-c b_{2}\right), \quad \delta_{0} \varepsilon_{4}=2\left(a_{1} b_{2}-c d\right)+b_{1} b_{2}-d^{2}, \\
\delta_{0} & =\left(2 a_{1}+b_{1}\right)\left(2 a_{2}+b_{2}\right)-(2 c+d)^{2} \equiv 4 \Delta_{0} d_{1} d_{2}>0, \\
\Delta_{0} & =m_{1} m_{3}-m_{2}^{2}, \quad m_{1}=l_{1}+\frac{l_{4}}{2}, \quad m_{2}=l_{2}+\frac{l_{5}}{2}, \quad m_{3}=l_{3}+\frac{l_{6}}{2}, \\
d_{1} & =\left(a_{1}+b_{1}\right)\left(a_{2}+b_{2}\right)-(c+d)^{2}>0, \quad d_{2}=a_{1} a_{2}-c^{2}>0, \\
l_{1} & =\frac{a_{2}}{d_{2}}, \quad l_{2}=-\frac{c}{d_{2}}, \quad l_{3}=\frac{a_{1}}{d_{2}}, \quad l_{1}+l_{2}=\frac{a_{2}+b_{2}}{d_{1}}, \\
l_{2} & +l_{5}=-\frac{c+d}{d_{1}}, \quad l_{3}+l_{6}=\frac{a_{1}+b_{1}}{d_{1}} .
\end{aligned}
$$


The expression for $\varepsilon^{T}$ can be rewritten as

$$
\varepsilon^{T}=-\frac{1}{2} l m^{-1}
$$

with

$$
l=\left[\begin{array}{ll}
l_{4}, & l_{5} \\
l_{5}, & l_{6}
\end{array}\right], \quad m=\left[\begin{array}{ll}
m_{1}, & m_{2} \\
m_{2}, & m_{3}
\end{array}\right],
$$

where $l_{j}(j=4,5,6)$ and $m_{j}(j=1,2,3)$ are defined by $(1.8)$.

Equation (1.7) is the basic homogeneous equation of statics of the elastic mixture theory in the complex form.

Applying the results obtained in [1], the vector $U$ can be represented as

$$
U=m \varphi_{*}(z)+\frac{l}{2} z \overline{\varphi_{*}^{\prime}(z)}+\overline{\psi_{*}(z)}
$$

where $m$ and $l$ are defined by $(1.10) ; \varphi_{*}(z)$ and $\psi_{*}(z)$ are arbitrary analytic vectors.

For the stress vector $T U$ we have

$$
\begin{aligned}
T U & =\left(\begin{array}{l}
(T U)_{2}-i(T U)_{1} \\
(T U)_{4}-i(T U)_{3}
\end{array}\right) \\
& =\frac{\partial}{\partial s(x)}\left[(A-2 E) \varphi_{*}(z)+B z \overline{\varphi_{*}^{\prime}(z)}+2 \mu \overline{\psi_{*}(z)}\right]
\end{aligned}
$$

where

$$
A=2 \mu m, \quad B=\mu l, \quad \mu=\left[\begin{array}{ll}
\mu_{1}, & \mu_{3} \\
\mu_{3}, & \mu_{2}
\end{array}\right], \quad \frac{\partial}{\partial s(x)}=n_{1} \frac{\partial}{\partial x_{2}}-n_{2} \frac{\partial}{\partial x_{1}},
$$

$n=\left(n_{1}, n_{2}\right)$ is an arbitrary unit vector.

The main goal of this investigation is to construct explicitly, in the form of absolutely and uniformly convergent series, the solutions of the basic boundary value problems (BVP) of the linear theory of elastic mixtures for an annulus. In particular, we will consider the so-called the first (i.e., the Dirichlet type) and the second (i.e., the Neumann type) BVPs with prescribed displacement and stress vectors on the boundary of the annulus, respectively. Similar problems in the classical theory of elasticity have been studied by Muskhelishvili in [4].

\section{Solution of the First Boundary Value Problem for an ANNULUS}

Let $(\rho, \varphi)$ be the polar coordinates in the plane $O x_{1} x_{2}$. Denote by $S_{j}(j=1,2)$ the circumference with center at the origin and radius $R_{j}, R_{2}>R_{1}$.

We are to find a regular solution of equation (1.7) in an annulus $R_{1}<\rho<R_{2}$ satisfying on $S_{1}$ and $S_{2}$ the Dirichlet type conditions:

$$
(U)_{\rho=R_{1}}=f(\varphi), \quad(U)_{\rho=R_{2}}=F(\varphi),
$$

where $f$ and $F$ are known vectors having the definite smoothness. 
We seek for a solution of the first (Dirichlet type) boundary value problem posed in the following form:

$$
\begin{aligned}
U= & h_{0}+\sum_{n=1}^{\infty}\left(\frac{R_{1}}{\rho}\right)^{n}\left(e^{-i n \varphi} h_{n}+e^{i n \varphi} h_{-n}\right) \\
& -\varepsilon^{T} \frac{\rho^{2}-R_{1}^{2}}{\rho^{2}} \sum_{n=1}^{\infty} n\left(\frac{R_{1}}{\rho}\right)^{n} e^{i(n+2) \varphi} \bar{h}_{n} \\
& +g_{0}+\sum_{n=1}^{\infty}\left(\frac{\rho}{R_{2}}\right)^{n}\left(e^{-i n \varphi} g_{n}+e^{i n \varphi} g_{-n}\right) \\
& -\varepsilon^{T} \frac{R_{2}^{2}-\rho^{2}}{R_{2}^{2}} \sum_{n=0}^{\infty}(n+2)\left(\frac{\rho}{R_{2}}\right)^{n} e^{-i n \varphi} g_{-(n+2)} \\
& +X \ln \frac{\rho}{R_{2}}-\frac{\varepsilon^{T}}{2} e^{2 i \varphi} \bar{X},
\end{aligned}
$$

where $h_{k}, g_{k}$ and $X$ are the sought for constant vectors.

The some of the first three terms in (2.2) is a regular solution of the first boundary value problem outside the circle of radius $R_{1}$, the sum of the next three terms is a regular solution inside the circle of radius $R_{2}$, while the last two terms are helpful in obtaining a solution of the first boundary value problem inside the annulus $R_{1}<\rho<R_{2}$.

Introduce the notation

$$
\lambda=\frac{R_{1}}{R_{2}}
$$

We have $0<\lambda<1, \ln \lambda<0$.

Passing to the limit in (2.2) when $\rho \rightarrow R_{1}$ and $\rho \rightarrow R_{2}$, we have

$$
\begin{aligned}
h_{0} & +\sum_{n=1}^{\infty}\left(e^{-i n \varphi} h_{n}+e^{i n \varphi} h_{-n}\right)+g_{0}+\sum_{n=1}^{\infty} \lambda^{n}\left(e^{-i n \varphi} g_{n}+e^{i n \varphi} g_{-n}\right) \\
& -\varepsilon^{T}\left(1-\lambda^{2}\right) \sum_{n=1}^{\infty}(n+2) \lambda^{n} e^{-2 n \varphi} \bar{g}_{-(n+2)} \\
& -2 \varepsilon^{T}\left(1-\lambda^{2}\right) \bar{g}_{-2}+X \ln \lambda-\frac{\varepsilon^{T}}{2} e^{2 i \varphi} \bar{X}=f(\varphi), \\
h_{0} & +\sum_{n=1}^{\infty} \lambda^{n}\left(e^{-i n \varphi} h_{n}+e^{i n \varphi} h_{-n}\right)+g_{0}+\sum_{n=1}^{\infty}\left(e^{-i n \varphi} g_{n}+e^{i n \varphi} g_{-n}\right) \\
& -\varepsilon^{T}\left(1-\lambda^{2}\right) \sum_{n=1}^{\infty} n \lambda^{n} e^{i(n+2) \varphi} \bar{h}_{n}-\frac{\varepsilon^{T}}{2} e^{2 i \varphi} \bar{X}=F(\varphi) .
\end{aligned}
$$


For the sought for coefficients $g_{n}, h_{n}, g_{-n}, h_{-n}, n=0,1,2, \ldots,(2.4)$ and $(2.5)$ yield the following equations:

$$
\begin{aligned}
& h_{0}+g_{0}-X \ln \lambda-2 \varepsilon^{T}\left(1-\lambda^{2}\right) \bar{g}_{-2}=f_{0}, \quad h_{0}+g_{0}=F_{0}, \\
& h_{-2}+\lambda^{2} g_{-2}-\frac{\varepsilon^{T}}{2} \bar{X}=f_{-2}, \quad \lambda^{2} h_{-2}+g_{-2}-\frac{\varepsilon^{T}}{2} \bar{X}=F_{-2}, \\
& h_{-1}+\lambda g_{-1}=f_{-1}, \quad \lambda h_{-1}+g_{-1}=F_{-1}, \\
& h_{n}+\lambda^{n} g_{n}-\varepsilon^{T}\left(1-\lambda^{2}\right)(n+2) \lambda^{n} \bar{g}_{-(n+2)}=f_{n}, \\
& \lambda^{n} h_{n}+g_{n}=F_{n}, \quad n \geq 1, \\
& \lambda^{n} h_{-n}+g_{-n}-\varepsilon^{T}\left(1-\lambda^{2}\right)(n-2) \lambda^{n-2} \bar{h}_{n-2}=F_{-n}, \\
& h_{-n}+\lambda^{n} g_{-n}=f_{-n}, \quad n \geq 3 .
\end{aligned}
$$

Here $f_{n}$ and $F_{n}, n=0, \pm 1, \pm 2, \ldots$, are the Fourier coefficients of the vectors $f$ and $F$ :

$$
f_{n}=\frac{1}{2 \pi} \int_{0}^{2 \pi} f(t) e^{i n t} d t, \quad F_{n}=\frac{1}{2 \pi} \int_{0}^{2 \pi} F(t) e^{i n t} d t .
$$

Let us first consider system (2.6). By elementary calculations we obtain

$$
\begin{aligned}
& \left(1-\lambda^{4}\right) h_{-2}=\frac{\varepsilon^{T}}{2}\left(1-\lambda^{2}\right) \bar{X}+f_{-2}-\lambda^{2} F_{-2}, \\
& \left(1-\lambda^{4}\right) g_{-2}=\frac{\varepsilon^{T}}{2}\left(1-\lambda^{2}\right) \bar{X}+F_{-2}-\lambda^{2} f_{-2} .
\end{aligned}
$$

Substituting the value $g_{-2}$ into (2.6), we have

$$
\left[E \ln \lambda-\left(\varepsilon^{T}\right)^{2} \frac{1-\lambda^{2}}{1+\lambda^{2}}\right] X=f_{0}-F_{0}+2 \varepsilon^{T} \frac{\bar{F}_{-2}-\lambda^{2} F_{-2}}{1+\lambda^{2}},
$$

where

$$
E=\left[\begin{array}{ll}
1, & 0 \\
0, & 1
\end{array}\right]
$$

Introduce the notation

$$
D_{0}=E \ln \lambda-\left(\varepsilon^{T}\right)^{2} \frac{1-\lambda^{2}}{1+\lambda^{2}}
$$

It is easy to show that

$$
\operatorname{det} D_{0}=\left(\ln \lambda-k_{1}^{2} \frac{1-\lambda^{2}}{1+\lambda^{2}}\right)\left(\ln \lambda-k_{2}^{2} \frac{1-\lambda^{2}}{1+\lambda^{2}}\right),
$$

where $k_{1}^{2}<1, k_{2}^{2}<1, k_{1}$ and $k_{2}$ are defined in [3] (formula (1.15)). It obviously follows that

$$
\operatorname{det} D_{0}>0
$$


From (2.6) we have

$$
\begin{aligned}
\bar{h}_{-2}= & \frac{1}{1-\lambda^{4}}\left\{\bar{f}_{-2}-\lambda^{2} \bar{F}_{-2}\right. \\
& \left.+\frac{1-\lambda^{2}}{2} \frac{\left[\varepsilon^{T} \ln \lambda-k_{1} k_{2}\left[\begin{array}{cc}
\varepsilon_{4}, & -\varepsilon_{3} \\
-\varepsilon_{2}, & \varepsilon_{1}
\end{array}\right] \frac{1-\lambda^{2}}{1+\lambda^{2}}\right]\left(f_{0}-F_{0}\right)}{\operatorname{det} D_{0}}\right\}, \\
\bar{g}_{-2}= & \frac{1}{1-\lambda^{4}}\left\{\bar{F}_{-2}-\lambda^{2} \bar{f}_{-2}\right. \\
& \left.+\frac{1-\lambda^{2}}{2} \frac{\left[\varepsilon^{T} \ln \lambda-k_{1} k_{2}\left[\begin{array}{cc}
\varepsilon_{4}, & -\varepsilon_{3} \\
-\varepsilon_{2}, & \varepsilon_{1}
\end{array}\right] \frac{1-\lambda^{2}}{1+\lambda^{2}}\right]\left(f_{0}-F_{0}\right)}{\operatorname{det} D_{0}}\right\} .
\end{aligned}
$$

Thus system (2.6) is solved.

Further, from (2.7) we obtain

$$
g_{-1}=\frac{F_{-1}-\lambda f_{-1}}{1-\lambda^{2}}, \quad h_{-1}=\frac{f_{-1}-\lambda F_{-1}}{1-\lambda^{2}}
$$

From (2.8) we have

$$
\left.\begin{array}{c}
\left(1-\lambda^{2 n}\right) h_{n}-\varepsilon^{T}\left(1-\lambda^{2}\right)(n+2) \lambda^{n} \bar{g}_{-(n+2)}=f_{n}-\lambda^{n} F_{n} \\
\left(1-\lambda^{2 n}\right) g_{n}+\varepsilon^{T}\left(1-\lambda^{2}\right)(n+2) \lambda^{2 n} \bar{g}_{-(n+2)}=F_{n}-\lambda^{n} f_{n}
\end{array}\right\} \quad n \geq 1,
$$

while (2.9) implies

$$
\left.\begin{array}{rl}
\left(1-\lambda^{2 n+4}\right) h_{-(n+2)} & +\varepsilon^{T}\left(1-\lambda^{2}\right) n \lambda^{2 n-2} \bar{h}_{n} \\
& =f_{-(n+2)}-\lambda^{n+2} F_{-(n+2)} \\
\left(1-\lambda^{2 n+4}\right) g_{-(n+2)} & -\varepsilon^{T}\left(1-\lambda^{2}\right) n \lambda^{n} \bar{h}_{n} \\
& =F_{-(n+2)}-\lambda^{n+1} f_{-(n+2)}
\end{array}\right\} \quad n \geq 1
$$

By the second equation we define $\bar{g}_{-(n+2)}$ and substitute it into the first equation in (2.12). We obtain

$$
\begin{aligned}
& {\left[\left(1-\lambda^{2 n}\right)\left(1-\lambda^{2 n+4}\right) E-\left(\varepsilon^{T}\right)^{2}\left(1-\lambda^{2}\right)^{2} n(n+2) \lambda^{2 n}\right] h_{n}} \\
& =\left(1-\lambda^{2 n+4}\right)\left(f_{n}-\lambda^{n} F_{n}\right) \\
& \quad+\varepsilon^{T}\left(1-\lambda^{2}\right)(n+2)\left(\bar{E}_{-(n+2)}-\lambda^{n+2} \bar{f}_{-(n+2)}\right), \quad n \geq 1 .
\end{aligned}
$$

Denote

$$
D_{n}=\left(1-\lambda^{2 n}\right)\left(1-\lambda^{2 n+4}\right) E-\left(\varepsilon^{T}\right)^{2}\left(1-\lambda^{2}\right)^{2} n(n+2) \lambda^{2 n} .
$$

After some calculations we find

$$
\begin{aligned}
\operatorname{det} D_{n}= & {\left[\left(1-\lambda^{2 n}\right)\left(1-\lambda^{2 n+4}\right)-k_{1}^{2}\left(1-\lambda^{2}\right)^{2} n(n+2) \lambda^{2 n}\right] } \\
& \times\left[\left(1-\lambda^{2 n}\right)\left(1-\lambda^{2 n+4}\right)-k_{2}^{2}\left(1-\lambda^{2}\right)^{2} n(n+2) \lambda^{2 n}\right]>0, \quad n \geq 1 .
\end{aligned}
$$

Moreover, $\lim _{n \rightarrow+\infty} D_{n}=1$. 
From (2.14) we obtain

$$
\begin{aligned}
h_{n}= & \frac{1}{\operatorname{det} D_{n}}\left[\left(1-\lambda^{2 n}\right)\left(1-\lambda^{2 n+4}\right) E\right. \\
& \left.-\left[\begin{array}{cc}
\varepsilon_{4}, & -\varepsilon_{3} \\
-\varepsilon_{2}, & \varepsilon_{1}
\end{array}\right]\left(1-\lambda^{2}\right)^{2} n(n+2) \lambda^{2 n}\right]\left[\left(1-\lambda^{2 n+4}\left(f_{n}-\lambda^{n} F_{n}\right)\right.\right. \\
& \left.+\varepsilon^{T}\left(1-\lambda^{2}\right)(n+2)\left(\bar{F}_{-(n+2)}-\lambda^{n+2} \bar{f}_{-(n+2)}\right)\right]>0, \quad n=1,2, \ldots
\end{aligned}
$$

Substituting the value $h_{n}$ into $(2.13)$, we uniquely define $\bar{g}_{-(n+2)}$ and $\bar{h}_{-(n+2)}$.

Thus we have uniquely defined all the sought for coefficients. Let us substitute the values of these coefficients into (2.2). Then we obtain a solution of the first boundary value problem in the form of a series. For these series together with their first derivatives to be absolutely and uniformly convergent it is sufficient that the functions $f^{\prime}(\varphi)$ and $F^{\prime}(\varphi)$ satisfy the Hölder condition with an exponent $\alpha>\frac{1}{2}$. Solutions obtained under such conditions are regular in an annulus.

Thus we have proved the following

Theorem 1. The Dirichlet type BVP (1.7), (2.1) is uniquely solvable in the class of regular vectors, and the solution is represented in the form of absolutely and uniformly convergent series (2.2), where the constant vectors $h_{k}$ and $g_{k}$ $(k=0, \pm 1, \ldots)$ solve the system of equations (2.6)-(2.9), if the boundary data $f$ and $F$ are from the space $C^{1, \alpha}$ with $\alpha>\frac{1}{2}$.

\section{Solution of the Second Boundary Value Problem for an ANNULUS}

In this paragraph we will construct an explicit solution of the boundary value problem for equation (1.7) when stresses are assumed to be given on the concentrical circumferences $S_{1}$ and $S_{2}$ :

$$
(T U)_{\rho=R_{1}}=f, \quad(T U)_{\rho=R_{2}}=F,
$$

where the vector $T U$ is defined by (1.11).

We seek the stress vector in the annulus $R_{1}<\rho<R_{2}$ in the following form:

$$
\begin{aligned}
T U= & \frac{R_{1}}{\rho} h_{0}+\sum_{n=1}^{\infty}\left(\frac{R_{1}}{\rho}\right)^{n+1}\left(e^{-i n \varphi} h_{n}+e^{i n \varphi} h_{-n}\right) \\
& +\frac{1}{\Delta_{2}}\left[\begin{array}{ll}
H_{1}, & H_{2} \\
H_{3}, & H_{4}
\end{array}\right] \frac{\rho^{2}-R_{1}^{2}}{\rho^{2}} \sum_{n=0}^{\infty}(n+2)\left(\frac{R_{1}}{\rho}\right)^{n+1} e^{i(n+1) \varphi} \bar{h}_{n} \\
& +\sum_{n=1}^{\infty}\left(\frac{\rho}{R_{2}}\right)^{n-1}\left(e^{-i n \varphi} g_{n}+e^{i n \varphi} h_{-n}\right) \\
& +\frac{1}{\Delta_{2}}\left[\begin{array}{ll}
H_{1}, & H_{2} \\
H_{3}, & H_{4}
\end{array}\right] \frac{R_{2}^{2}-\rho^{2}}{R_{2}^{2}} \sum_{n=1}^{\infty} n\left(\frac{\rho}{R_{2}}\right)^{n-1} e^{-i n \varphi} \bar{g}_{-(n+2)},
\end{aligned}
$$

where $\Delta_{2}=\operatorname{det}(A-2 E)>0$ and $H_{1}, H_{2}, H_{3}, H_{4}$ are the definite complex constants [3]. 
Here $h_{0}, h_{n}, h_{-n}, g_{n}, g_{-n}(n=1,2, \ldots)$ are the constant vectors to be found. Using the boundary conditions (3.1), we have

$$
\begin{aligned}
h_{0} & +\sum_{n=1}^{\infty}\left(e^{-i n \varphi} h_{n}+e^{i n \varphi} h_{-n}\right)+\sum_{n=1}^{\infty} \lambda^{n-1}\left(e^{-i n \varphi} g_{n}+e^{i n \varphi} g_{-n}\right) \\
& +\frac{1}{\Delta_{2}}\left[\begin{array}{ll}
H_{1}, & H_{2} \\
H_{3}, & H_{4}
\end{array}\right]\left(1-\lambda^{2}\right) \sum_{n=1}^{\infty} n \lambda^{n-1} e^{-i n \varphi} \bar{g}_{-(n+2)}=f(\varphi), \\
\lambda h_{0}+ & \sum_{n=1}^{\infty} \lambda^{n+1}\left(e^{-i n \varphi} h_{n}+e^{i n \varphi} h_{-n}\right)+\sum_{n=1}^{\infty}\left(e^{-i n \varphi} g_{n}+e^{i n \varphi} g_{-n}\right) \\
& +\frac{1}{\Delta_{2}}\left[\begin{array}{ll}
H_{1}, & H_{2} \\
H_{3}, & H_{4}
\end{array}\right]\left(1-\lambda^{2}\right) \sum_{n=1}^{\infty}(n+2) \lambda^{n+1} e^{i(n+2) \varphi} \bar{h}_{n}=F(\varphi),
\end{aligned}
$$

where $f$ and $F$ are the given vectors.

Using (3.1) and standard approach for the coefficients $h_{0}, h_{n}, h_{-n}, g_{n}, g_{-n}$ we obtain the algebraic equations

$$
\begin{aligned}
& h_{0}=f_{0}, \quad \lambda h_{0}=F_{0} \text {, } \\
& h_{-1}+g_{-1}=f_{-1}, \quad \lambda^{2} h_{-1}+g_{-1}=F_{-1} \text {, } \\
& \left.\begin{array}{l}
h_{n}+\lambda^{n-1} g_{n}+\frac{1}{\Delta_{2}}\left[\begin{array}{ll}
H_{1}, & H_{2} \\
H_{3}, & H_{4}
\end{array}\right]\left(1-\lambda^{2}\right) n \lambda^{n-1} \bar{g}_{-(n+2)}=f_{n} \\
\lambda^{n+1} h_{n}+g_{n}=F_{n}
\end{array}\right\} n \geq 1, \\
& \left.\begin{array}{l}
\lambda^{n+1} h_{-n}+g_{-n}+\frac{n}{\Delta_{2}}\left[\begin{array}{ll}
H_{1}, & H_{2} \\
H_{3}, & H_{4}
\end{array}\right] \lambda^{n-1} \bar{h}_{n-1}=F_{n} \\
h_{-n}+\lambda^{n-1} g_{-n}=f_{-n}
\end{array}\right\} n \geq 2,
\end{aligned}
$$

where $f_{n}$ and $F_{n}(n=0, \pm 1, \pm 2, \ldots)$ are the Fourier coefficients of the functions $f$ and $F$, respectively.

From (3.3) it follows that $\lambda f_{0}=F_{0}$, i.e., $R_{1} f_{0}=R_{2} F_{0}$, which fact coincides with the stress equilibrium condition (the principal stress vector is equal to zero). Therefore we can write

$$
h_{0}=\frac{F_{0}}{\lambda}=f_{0}
$$

Let us rewrite (3.4) as

$$
h_{-1}+g_{-1}=f_{-1}, \quad R_{1}^{2} h_{-1}+R_{2}^{2} g_{-1}=R_{2}^{2} F_{-1} .
$$

Since the principal stress moment is to be equal to zero, i.e., $R_{1}^{2} f_{-1}=R_{2}^{2} F_{-1}$, we obtain

$$
g_{-1}=0, \quad h_{-1}=f_{-1}=\frac{R_{2}^{2}}{r_{1}^{2}} F_{-1}
$$

Thus we have defined $g_{-1}$ and $h_{-1}$. 
Using equation (3.6), we obtain

$$
\begin{aligned}
h_{-1} & =\frac{1}{1-\lambda^{4}}\left[f_{-2}-\lambda F_{-2}+\frac{2}{\Delta_{2}}\left[\begin{array}{ll}
H_{1}, & H_{2} \\
H_{3}, & H_{4}
\end{array}\right]\left(1-\lambda^{2}\right) \lambda^{2} f_{0}\right], \\
g_{-1} & =\frac{1}{1-\lambda^{4}}\left[F_{-2}-\lambda^{3} f_{-2}-\frac{2}{\Delta_{2}}\left[\begin{array}{ll}
H_{1}, & H_{2} \\
H_{3}, & H_{4}
\end{array}\right]\left(1-\lambda^{2}\right) \lambda f_{0}\right] .
\end{aligned}
$$

From (3.5) and (3.6) we have

$$
\begin{aligned}
& \left.\begin{array}{l}
\left(1-\lambda^{2 n}\right) h_{n}+\frac{n}{\Delta_{2}}\left[\begin{array}{ll}
H_{1}, & H_{2} \\
H_{3}, & H_{4}
\end{array}\right]\left(1-\lambda^{2}\right) \lambda^{n-1} \bar{g}_{-(n+2)}=f_{n}-\lambda^{n-1} F_{n} \\
\left(1-\lambda^{2 n}\right) g_{n}-\frac{n}{\Delta_{2}}\left[\begin{array}{ll}
H_{1}, & H_{2} \\
H_{3}, & H_{4}
\end{array}\right]\left(1-\lambda^{2}\right) \lambda^{2 n} \bar{g}_{-(n+2)}=F_{n}-\lambda^{n+1} f_{n}
\end{array}\right\} n \geq 1, \\
& \left.\left(1-\lambda^{2 n}\right) h_{-n}-\frac{n}{\Delta_{2}}\left[\begin{array}{ll}
H_{1}, & H_{2} \\
H_{3}, & H_{4}
\end{array}\right]\left(1-\lambda^{2}\right) \lambda^{2 n-2} \bar{h}_{n-2}=f_{-n}-\lambda^{n-1} F_{-n}\right) \\
& \left.\left(1-\lambda^{2 n}\right) g_{-n}-\frac{n}{\Delta_{2}}\left[\begin{array}{ll}
H_{1}, & H_{2} \\
H_{3}, & H_{4}
\end{array}\right]\left(1-\lambda^{2}\right) \lambda^{n-1} \bar{h}_{n-1}=F_{-n}-\lambda^{n+1} f_{-n}\right\} n \geq 3 \text {. }
\end{aligned}
$$

From the second equation of (3.8) we can write

$$
\begin{aligned}
&\left\{\left(1-\lambda^{2 n}\right)\left(1-\lambda^{2 n+4}\right) E-\frac{1}{\Delta_{2}^{2}}\left[\begin{array}{ll}
H_{1}, & H_{2} \\
H_{3}, & H_{4}
\end{array}\right]\left(1-\lambda^{2}\right)^{2} n(n+2) \lambda^{2 n}\right\} h_{n} \\
&=-\frac{1}{\Delta_{2}}\left[\begin{array}{cc}
H_{1}, & H_{2} \\
H_{3}, & H_{4}
\end{array}\right]\left(1-\lambda^{2}\right) n\left(\bar{F}_{-(n+2)}-\lambda^{n+3} \bar{f}_{-(n+2)}\right)+\left(1-\lambda^{2 n+4}\right)\left(f_{n}-\lambda^{n-1} F_{n}\right),
\end{aligned}
$$

where $E$ is the unit matrix.

Thus we have obtained one vector relation for defining $h_{n}$. Denote

$$
D_{n}=\left(1-\lambda^{2 n}\right)\left(1-\lambda^{2 n+4}\right) E-\frac{1}{\Delta_{2}^{2}}\left[\begin{array}{ll}
H_{1}, & H_{2} \\
H_{3}, & H_{4}
\end{array}\right]\left(1-\lambda^{2}\right)^{2} n(n+2) \lambda^{2 n} .
$$

After some calculations we find

$$
\begin{aligned}
\operatorname{det} D_{n}= & {\left[\left(1-\lambda^{2 n}\right)\left(1-\lambda^{2 n+4}\right)-\left(1-\lambda^{2}\right) n(n+2) \lambda^{2 n}\right]\left[\left(1-\lambda^{2 n}\right)\left(1-\lambda^{2 n+4}\right)\right.} \\
& \left.-\left(1-\lambda^{2}\right) n(n+2) \lambda^{2 n}-\frac{4 \lambda_{5}\left(a_{0} b+b_{0} \Delta_{1}\right)\left(1-\lambda^{2}\right)^{2} n(n+2) \lambda^{2 n}}{d_{2} d_{1}^{2}}\right],
\end{aligned}
$$

where $\lambda_{5}<0, a_{0}=a_{1}+a_{2}+2 c \equiv \mu_{1}+\mu_{2}+2 \mu_{3}>0, b_{0}=b_{1}+b_{2}+2 d=$ $b_{1}-\lambda_{5}+b_{2}-\lambda_{5}+2\left(d+\lambda_{5}\right)>0, \Delta_{1}=\mu_{1} \mu_{2}-\mu_{3}^{2}>0, d_{2}=a_{1} a_{2}-c^{2}=\Delta_{1}-\lambda_{5} a_{0}$, $d_{1}=\left(a_{1}+b_{1}\right)\left(a_{2}+b_{2}\right)-(c+d)^{2} \equiv \Delta_{1}+a+b, a=\mu_{1}\left(b_{2}-\lambda_{5}\right)+\mu_{2}\left(b_{1}-\lambda_{5}\right)-$ $2 \mu_{3}\left(d+\lambda_{5}\right)>0, b=\left(b_{1}-\lambda_{5}\right)\left(b_{2}-\lambda_{5}\right)-\left(d+\lambda_{5}\right)^{2}>0$.

We prove

$$
\left(1-\lambda^{2 n}\right)\left(1-\lambda^{2 n+4}\right)-\left(1-\lambda^{2}\right) n(n+2) \lambda^{2 n}>0, \quad n \geq 1,
$$

and therefore $\operatorname{det} D_{n}>0, n \geq 1$. Moreover, $\lim _{n \rightarrow+\infty} D_{n}=1$.

Thus

$$
h_{n}=\frac{1}{\operatorname{det} D_{n}}\left[\left(1-\lambda^{2 n}\right)\left(1-\lambda^{2 n+4}\right) E-\frac{1}{\Delta_{2}^{2}}\left[\begin{array}{ll}
H_{1}, & H_{2} \\
H_{3}, & H_{4}
\end{array}\right]^{2}\left(1-\lambda^{2}\right)^{2} n(n+2) \lambda^{2 n}\right]
$$




$$
\begin{aligned}
& \times\left[-\frac{n}{\Delta_{2}}\left[\begin{array}{ll}
H_{1}, & H_{2} \\
H_{3}, & H_{4}
\end{array}\right]\left(1-\lambda^{2}\right)^{2} \lambda^{n+1}\left(\bar{F}_{-(n+2)}-\lambda^{n+3} \bar{f}_{-(n+2)}\right)\right. \\
& +\left(1-\lambda^{2 n+4}\right)\left(f_{n}-\lambda^{n-1} F_{n}\right) .
\end{aligned}
$$

Substituting the expression of $h_{n}$ into (3.8), we can define $g_{-n}$ and $h_{-n}$ for any $n \geq 3$. Finally, using (3.7) we define $g_{n}$ for $n \geq 1$.

Thus we have defined all the unknown coefficients $g_{n}, h_{n}, g_{-n}, h_{-n}$.

To conclude, note that all sought for coefficients are defined uniquely if the principal vector and the principal moment of stresses are equal to zero.

Substituting the defined coefficients into formula (3.1), we obtain the stress vector in the form of series for the annulus $R_{1}<\rho<R_{2}$. Series (3.2) is absolutely and uniformly convergent if the given vectors $f$ and $F$ satisfy the Hölder condition with an exponent $\alpha>\frac{1}{2}$.

Thus we have proved the following

Theorem 2. The Neumann type BVP (1.7), (3.1) is solvable and the corresponding stress vector is represented in the form of absolutely and uniformly convergent series (3.2), where the constant vectors $h_{k}$ and $g_{k}(k=0, \pm 1, \ldots)$ solve the system of equations (3.3)-(3.6), if the boundary data $f$ and $F$ belong to the space $C^{0, \alpha}$ with $\alpha>\frac{1}{2}$ and the corresponding principal (resultant) vector and moment are equal to zero.

\section{REFERENCES}

1. M. Basheleishvili, Analogues of the general Kolosov-Muskhelishvili representation formulas and Cauchy-Riemann conditions of the theory of elastic mixtures. Georgian Math. J. 4(1997), No. 3, 223-242.

2. M. Basheleishvili and K. Svanadze, A new method of solving the basic plane boundary value problems of statics of the elastic mixture theory. Georgian Math. J. 8(2001), No. 3, 427-446.

3. M. BAsheleishvili, Application of analogues of general Kolosov-Muskhelishvili representations in the theory of elastic mixtures. Georgian Math. J. 6(1999), No. 1, 1-18.

4. N. I. Muskhelishvili, Some basic problems of the mathematical theory of elasticity. (Russian) 5th ed. Nauka, Moscow, 1966; 2nd English ed.: P. Noordhoff, Ltd., Groningen, 1963.

(Received 27.05.2003)

Author's address:

I. Vekua Institute of Applied Mathematics

I. Javakhishvili Tbilisi State University

2, University St., Tbilisi 0143

Georgia 